\title{
Cooperação internacional: doações de medicamentos realizadas pelo governo brasileiro de 2005 a 2016
}

\author{
Alane Andrelino Ribeiro, ${ }^{1}$ Evandro de Oliveira Lupatini ${ }^{1}$ \\ e Diana Graziele dos Santos ${ }^{1}$
}

Como citar Ribeiro AA, Lupatini EO, Santos DG. Cooperação internacional: doações de medicamentos realizadas pelo governo brasileiro de 2005 a 2016. Rev Panam Salud Publica. 2018;42:e67. https://doi. org/10.26633/RPSP.2018.67

RESUMO

Objetivo. Fazer um levantamento acerca das doações internacionais de medicamentos pelo governo brasileiro de 2005 a 2016, identificando o número de unidades de medicamentos doadas e o montante financeiro que esse volume representa.

Métodos. Estudo descritivo e exploratório utilizando análise de dados secundários disponíveis no Sistema de Gestão de Materiais do Ministério da Saúde (SISMAT). Foram levantadas informações sobre país solicitante, ano da doação, medicamento, número de unidades farmacêuticas doadas e valor correspondente (em reais).

Resultados. No período estudado, 66554892 unidades de medicamentos foram doadas, correspondendo a 84371 308,72 reais (25 505 235,70 dólares). Foram doados medicamentos para Angola, Benin, Bolívia, Burkina Faso, Cabo Verde, Colômbia, Costa do Marfim, Cuba, El Salvador, Equador, Guatemala, Guiana, Guiné Bissau, Haiti, Honduras, Líbia, Moçambique, Nicarágua, Paraguai, Peru, República Dominicana, São Tomé e Príncipe, Síria e Suriname, países do Mercado Comum e Comunidade do Caribe (CARICOM) e Organização PanAmericana da Saúde (OPAS). Não foi detectado nenhum padrão de distribuição em termos quantitativos. Picos no número de medicamentos doados ocorreram em 2008, 2011 e 2012, com maior número de países contemplados em 2006 e 2012 (13 e 14 países, respectivamente). Foram doados medicamentos para HIV/Aids, malária, leishmaniose, diabetes, cólera, esquistossomose, tuberculose, gripe influenza, doenças oportunistas, imunizações, suporte nutricional e calamidade pública.

Conclusões. Em grande medida, os medicamentos doados foram antimicrobianos utilizados para tratamento de doenças tropicais negligenciadas. Estudos adicionais são sugeridos para correlacionar intervenções de saúde pública com as doações de medicamentos, como forma de promover o desenvolvimento econômico sustentável dos países beneficiários.

Palavras-chave Cooperação internacional; doações; medicamentos essenciais; assistência à saúde; Brasil.

Entre as políticas externas prioritárias do Brasil, como parte de um movimento de expansão diplomática, estão as

\footnotetext{
Ministério da Saúde, Departamento de Assistência Farmacêutica e Insumos Estratégicos, Brasília (DF), Brasil. Correspondência: Alane Andrelino Ribeiro, alane.andrelino@gmail.com
}

intervenções de cooperação em saúde. Tais intervenções têm potencial para desafiar modelos estabelecidos e apresentar alternativas aos projetos tradicionais de cooperação (1), fortalecendo os sistemas de saúde centrados nas pessoas e na solidariedade social (2).
Se, de um lado, o acesso a medicamentos é fundamental no cuidado à saúde, de outro tornou-se motivo de preocupação devido à dificuldade para sua garantia. As causas para essa dificuldade compreendem desde a falta de pessoal qualificado para a gestão da assistência 
farmacêutica até limitações financeiras decorrentes do aumento dos gastos em saúde. Soma-se a essas dificuldades o fato de os medicamentos serem considerados pelo mercado como produtos capazes de gerar lucros expressivos, sobretudo com as patentes sobre inovações terapêuticas, regulamentadas pelo Tratado de Propriedade Intelectual $(3,4)$, diretamente relacionado à transferência de tecnologia de inovações na área de medicamentos e à priorização das pesquisas subsequentes (5).

No tocante à produção pública de medicamentos pelo Brasil, são pontos de destaque a importância do domínio tecnológico e os desafios da internalização da produção. Nesse sentido, visando a assegurar o acesso a medicamentos de importância estratégica para a população, o governo brasileiro instituiu diversas políticas para garantir a autonomia na produção nacional de medicamentos, buscando dirimir vulnerabilidades frente à dependência da produção tecnológica externa (6-8).

Atualmente, segundo dados do Ministério da Saúde, o Brasil conta com 31 instituições públicas oficiais dedicadas à produção de medicamentos, vacinas e produtos para saúde. Esse número destaca o potencial produtor do país para abastecimento próprio de alguns medicamentos necessários à população, como também para doação a outros países. Cita-se o caso do medicamento benznidazol, utilizado no tratamento da doença de Chagas e produzido apenas por um laboratório brasileiro (Laboratório Farmacêutico do Estado de Pernambuco, Lafepe) na região das Américas (9).

Ainda no contexto do acesso, as compras governamentais também se configuram como desafio e oportunidade para prover medicamentos a um custo menor, tirando proveito do poder de compra em larga escala do Estado. No âmbito do Mercado Comum do Sul (Mercosul), a iniciativa de compra conjunta de medicamentos de alto custo é um marco dos vínculos de integração e solidariedade em matéria de saúde que têm vigência nos países membros do Mercosul e da União de Nações SulAmericanas (UNASUL). Por exemplo, em 2015, essa iniciativa alcançou descontos de até $83 \%$ na aquisição de medicamentos para o tratamento da Aids (10).

A solicitação de doação de medicamentos geralmente é realizada pelo país solicitante à embaixada do Brasil naquele país. Por sua vez, a embaixada repassa a solicitação ao Ministério das Relações Exteriores do Brasil. Essa solicitação é então processada e encaminhada ao Ministério da Saúde brasileiro para tomada das providências cabíveis, em consonância com as ações de cooperação técnica internacional em saúde presentes nos planos plurianuais do Brasil especificamente nos quadriênios de 2004 a 2007, 2008 a 2011, 2012 a 2015 e 2016 a 2019, visando à solidariedade global com responsabilidade compartilhada (11).

Acerca da cooperação em saúde, uma recente publicação aponta que:

(...) o Brasil desponta, aos olhos de outros países em desenvolvimento, como uma esperança na cooperação Sul-Sul, já que dispõe de políticas públicas bem-sucedidas, particularmente na área da saúde, que podem oferecer alternativas eficazes a serem compartilhadas para a melhoria dos sistemas de saúde de países parceiros e para a saúde de suas populações (12, p. 249).

Em meio a projetos como a Cooperação Tripartite Brasil-Haiti-Cuba $(13,14)$, iniciada por ocasião do terremoto de 2010 no Haiti, é importante ressaltar que as cooperações são também um instrumento de inserção política no âmbito internacional. Isso ocorre especialmente no âmbito da cooperação Sul-Sul, na qual países compartilham capacidades especializadas e experiências exitosas em uma relação mais horizontal, consensual e com maior potencial de equidade do que na cooperação Norte-Sul $(15,16)$. Cabe relembrar que a cooperação brasileira em saúde é baseada nos princípios de cooperação entre os povos para o progresso da humanidade, respeito à soberania nacional, à independência econômica, aos direitos iguais e à não intervenção nos assuntos domésticos das nações, horizontalidade nas ações de cooperação, respeito à diversidade cultural e sustentabilidade das ações (17). Podem ser citadas como exemplo as ações de cooperação Brasil-Moçambique, tais como o projeto de implantação da fábrica de medicamentos antirretrovirais (18), conhecida como Sociedade Moçambicana de Medicamentos (SMM), nas quais desdobraram-se desafios específicos da cooperação Sul-Sul, tais como a responsabilidade mútua e a não condicionalidade (19).

Apesar desses avanços na doação internacional de medicamentos, ainda há escassez de informações descritivas em âmbito brasileiro, sobretudo quanto a sua efetividade e à ampliação do acesso a medicamentos nos países receptores (20). No bojo das ações de cooperação, que tipo e que quantidade de medicamentos o Brasil tem doado? Quais foram os países receptores? Pensando nessas questões, o objetivo do presente artigo foi fazer um levantamento acerca das doações internacionais de medicamentos pelo governo brasileiro de 2005 a 2016, identificando inclusive o número de unidades de medicamentos doadas e o montante financeiro que esse volume representa, com base nas distribuições realizadas pela Central de Abastecimento Farmacêutico do Ministério da Saúde.

\section{MATERIAIS E MÉTODOS}

Trata-se de um estudo descritivo e exploratório, que analisou dados secundários relativos à representação financeira dos medicamentos doados internacionalmente pelo Brasil de 2005 a 2016 por meio do Central de Abastecimento Farmacêutico (CAF) do Ministério da Saúde do Brasil. Para tal, foram coletados os dados disponíveis no Sistema de Gestão de Materiais do Ministério da Saúde (SISMAT), acessado através do Sistema de Controle de Acesso Web do Ministério da Saúde (www.saude.gov.br/scaweb), referentes às despesas e distribuições anuais de medicamentos armazenados no almoxarifado central do Ministério da Saúde (21). Os dados foram coletados por meio de pesquisa documental, mediante acesso direto ao sistema em questão.

Um instrumento próprio foi desenvolvido para a coleta dos seguintes dados: país solicitante, ano da doação, medicamento, quantidade doada (em número de unidades farmacêuticas) e valor correspondente (expresso em reais). Os medicamentos foram também agrupados de acordo com a finalidade terapêutica ou programas estratégicos do SUS, que contemplam o tratamento de doenças e agravos de perfil endêmico, com importância epidemiológica, impacto socioeconômico ou que acometem populações vulnerabilizadas. A opção pelo recorte temporal a partir do ano 2005 deve-se ao fato de que, antes desse ano, os dados não eram registrados pelo Ministério da Saúde no SISMAT.

Todos os valores foram atualizados de acordo com a variação média anual do Índice de Preços ao Consumidor Amplo (IPCA), do Instituto Brasileiro de Geografia e Estatística (IBGE) (22). Esse índice foi escolhido por ser o utilizado pelo 
Governo Federal para atualização de preços. Após atualização, os preços foram convertidos para dólares americanos, a partir da taxa de câmbio praticada pelo Banco Central do Brasil em 31 de dezembro de 2017 ( $\mathrm{R} \$ 1,00=\mathrm{US} \$$ 0,3022975). Os dados foram tabulados e organizados em planilhas do programa Microsoft Office Excel 2013. Utilizou-se a estatística descritiva para organizar, resumir e descrever o conjunto de dados coletado referente às doações de medicamentos.

Este estudo está estruturado conforme os marcos da cooperação técnica internacional, em suas duas vertentes: a cooperação horizontal (ou Sul-Sul) e a cooperação recebida do exterior (bilateral ou multilateral) nas dimensões de diplomacia e cooperação em saúde (23), no âmbito da localização espacial e geopolítica dos países receptores de medicamentos doados pelo governo brasileiro.

\section{RESULTADOS}

No período de 2005 a 2016, foram doadas 66554892 unidades de medicamentos, que somam um valor de R\$ 84371 308,72 (US\$ 25505 235,70). As doações foram realizadas a 24 países: Angola, Benin, Bolívia, Burkina Faso, Cabo Verde, Colômbia, Costa do Marfim, Cuba, El Salvador, Equador, Guatemala, Guiana, Guiné Bissau, Haiti, Honduras, Líbia, Moçambique, Nicarágua, Paraguai, Peru, República Dominicana, São Tomé e Príncipe, Síria e Suriname. Também receberam doações os países do Mercado Comum e Comunidade do Caribe (CARICOM) e a Organização PanAmericana da Saúde (OPAS). A doação para a OPAS ocorre quando a execução e a gestão de projetos de cooperação em saúde são realizadas por intermédio dessa organização.

Os medicamentos doados (Apêndice) destinam-se, sobretudo, ao tratamento de HIV/Aids, malária, leishmaniose, diabetes, cólera, esquistossomose, tuberculose, influenza, doenças oportunistas, imunização e nutrição e ao atendimento de situações de calamidade pública. Em geral, são doenças ou agravos negligenciados, relacionados à pobreza, para os quais o custo do tratamento medicamentoso é baixo, excetuando-se os medicamentos destinados ao tratamento de doenças hematológicas e de HIV / Aids.

A figura 1 apresenta a relação entre o número doado de unidades de

FIGURA 1. Número de unidades farmacêuticas doadas pelo governo brasileiro de 2005 a 2016 em relação ao número de países atendidos

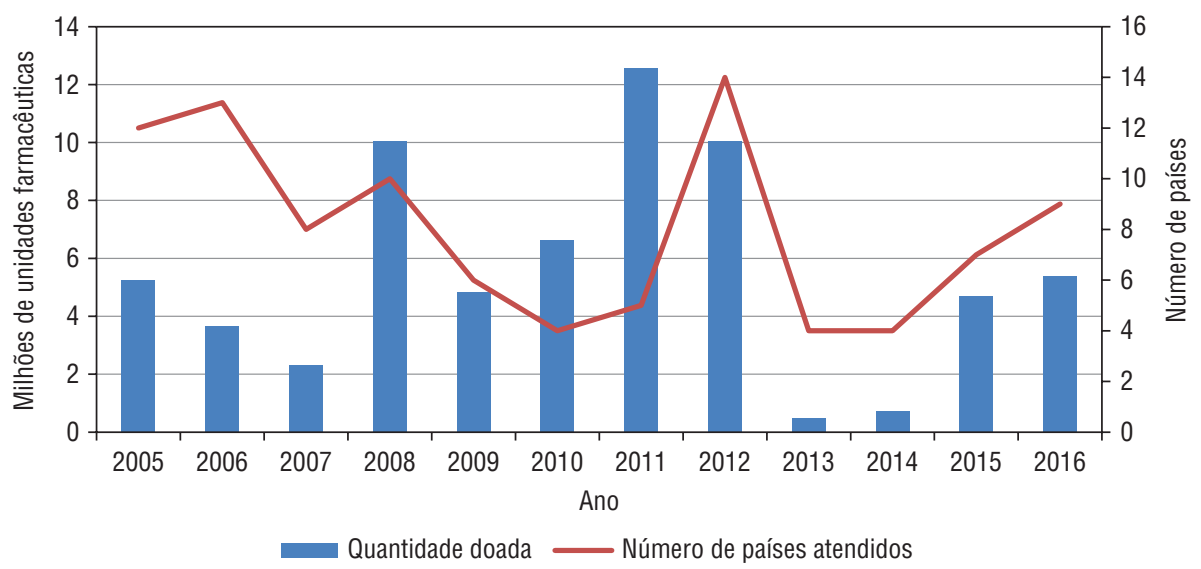

Fonte: Sistema de Gestão de Materiais do Ministério da Saúde (SISMAT).

FIGURA 2. Representação financeira ${ }^{a}$ e número de unidades farmacêuticas doadas pelo governo brasileiro no período de 2005 a 2016

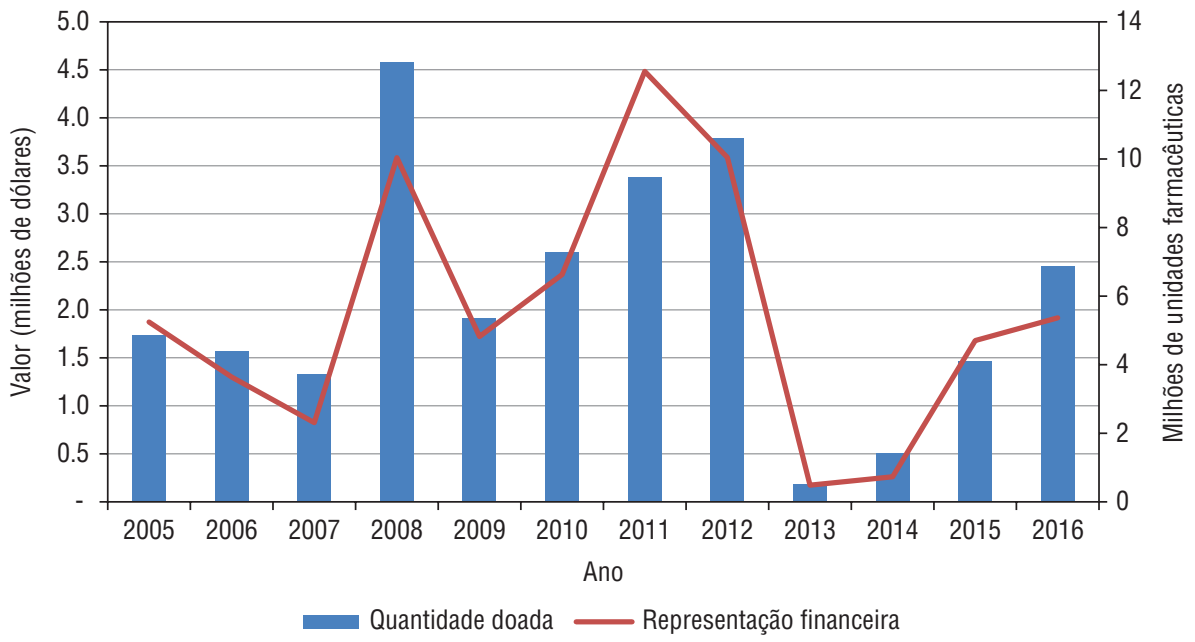

Fonte: Sistema de Gestão de Materiais do Ministério da Saúde (SISMAT). a Taxa de câmbio em dezembro de 2017: R\$1,00 = US\$ 0,3022975.

medicamentos e o número de países que as receberam. Observam-se picos no número de medicamentos doados em 2008, 2011 e 2012, com maior número de países contemplados em 2006 e 2012 (13 e 14 países, respectivamente). Os anos de 2013 e 2014 apresentaram uma redução importante no quantitativo distribuído. Essa redução resultou, possivelmente, da menor solicitação internacional nesse período, bem como de outros fatores, como a alteração de esquemas terapêuticos locais.

Ao longo dos anos avaliados, verificou-se que não há um padrão de distribuição anual. Em consulta ao site do Ministério da Saúde, não foi possível identificar a existência de um protocolo definido com atribuição de critérios para doação de medicamentos.
Quanto à relação entre o valor expresso em reais e o número de unidades farmacêuticas doadas de 2005 a 2016, a figura 2 revela que ambas seguem mesmo padrão de variação, sendo $\mathrm{R} \$ 1,26$ (US\$ 0,38) o valor unitário médio por unidade farmacêutica. A figura 3 mostra o número médio de unidades medicamentosas doadas de 2005 a 2016 e o número de países contemplados de acordo com a doença tratada. Como mostra a figura, os medicamentos para $\mathrm{HIV}$ / Aids foram os mais doados. Além disso, os medicamentos para HIV / Aids contemplaram 18 países e os medicamentos para tratamento de malária, 8 países no período. Vale notar que os medicamentos para tratamento de malária são produzidos prioritariamente 
por laboratórios públicos, como o Laboratório Farmacêutico Federal Farmanguinhos, da Fundação Oswaldo Cruz (Fiocruz), sendo que outra parte é adquirida pelo Brasil via OPAS.

No que se refere aos antirretrovirais (figura 4), verificou-se que os medicamentos mais doados durante o período foram lamivudina $150 \mathrm{mg}+$ zidovudina $300 \mathrm{mg}$, nevirapina $200 \mathrm{mg}$ e sulfato de indinavir $400 \mathrm{mg}$. Esses três medicamentos foram produzidos por laboratórios públicos brasileiros, o que mostra a relevância da produção pública de medicamentos também no âmbito da cooperação internacional. Os anos com maior doação de antirretrovirais foram 2008, 2010 e 2012, o que novamente demonstra a falta de linearidade nas doações de antirretrovirais distribuídos a partir da CAF do Ministério da Saúde ao longo do período analisado.

\section{DISCUSSÃO}

O presente estudo mostra que os medicamentos doados pelo governo brasileiro foram destinados a países de baixa e média renda, demonstrando cooperação focada no eixo Sul-Sul. É importante destacar que, no mesmo período estudado, a CAF do Ministério da Saúde distribuiu em território nacional 11548036876 unidades de medicamentos, representando R R $35 \quad 275 \quad 928 \quad 228,00$ (US\$ 10663824 914,00). Sendo assim, as doações internacionais representaram 0,576\% das unidades de medicamentos distribuídas nacionalmente, correspondendo a $0,003 \%$ em termos financeiros expresso em reais. Apesar de ser este um montante financeiro extremamente baixo frente ao total distribuído pelo governo brasileiro, considerando o próprio país e as doações, infere-se que as doações tenham produzido impacto significativo no país receptor, na medida em que contribuíram para o atendimento das necessidades de saúde de sua população.

Os antirretrovirais e antimaláricos foram os medicamentos mais doados no período estudado. Isso pode estar relacionado a elementos como a inclusão do combate ao HIV / Aids e à malária como Objetivo de Desenvolvimento do Milênio (24); a existência de projetos brasileiros de cooperação internacional em HIV / Aids (11); o arcabouço jurídico-normativo que garante o tratamento antirretroviral no Brasil, especialmente a lei 9313 de 1996, tornando o Brasil um país

FIGURA 3. Média total de unidades farmacêuticas doadas pelo Brasil e número de países beneficiados de acordo com a condição de saúde para o período de 2005 a 2016

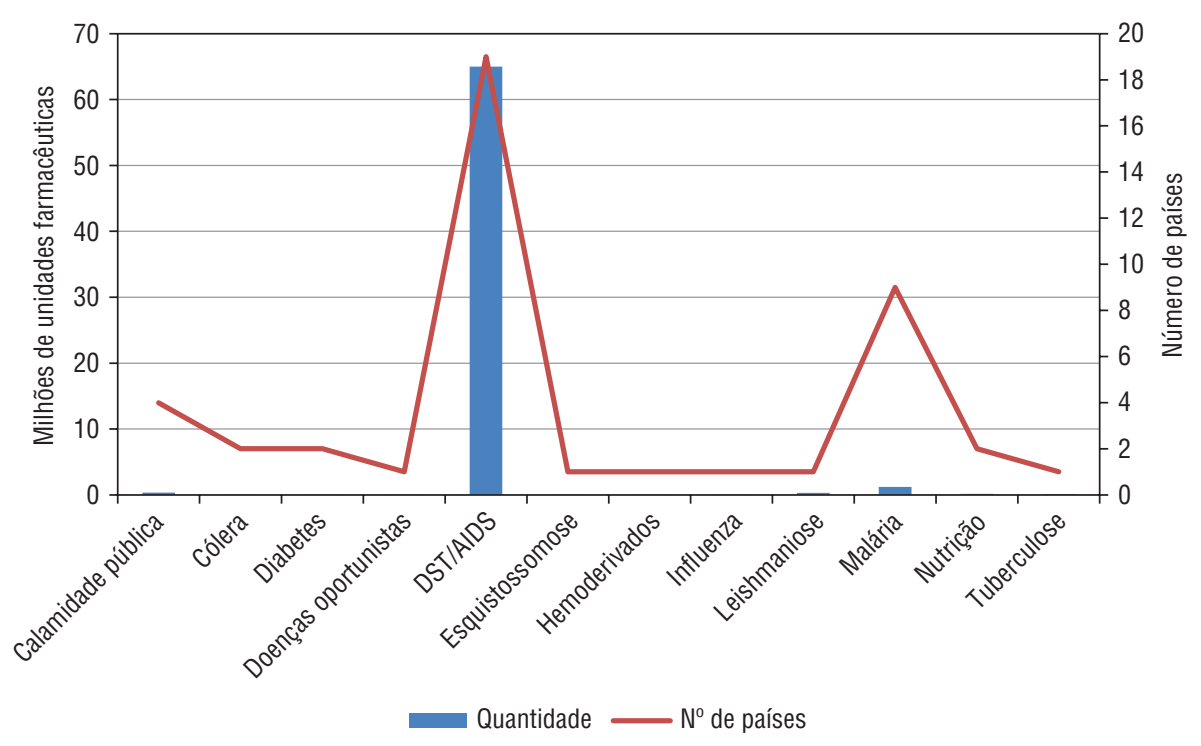

\begin{tabular}{|lrc|}
\hline Programa de saúde & Quantidade & $\mathbf{N}^{0}$ de países \\
\hline Calamidade pública & 346050 & 4 \\
Cólera & 50750 & 2 \\
Diabetes & 13000 & 2 \\
Doenças oportunistas & 2152 & 1 \\
HIV/Aids & 64338591 & 18 \\
Esquistossomose & 500 & 1 \\
Hemoderivados & 1410 & 1 \\
Influenza & 5300 & 1 \\
Leishmaniose & 300000 & 1 \\
Malária & 1184835 & 8 \\
Nutrição & 157000 & 2 \\
Tuberculose & 155304 & 1 \\
\hline
\end{tabular}

Fonte: Sistema de Gestão de Materiais do Ministério da Saúde (SISMAT).

FIGURA 4. Número de antirretrovirais doados pelo Brasil no período de 2005 a 2016

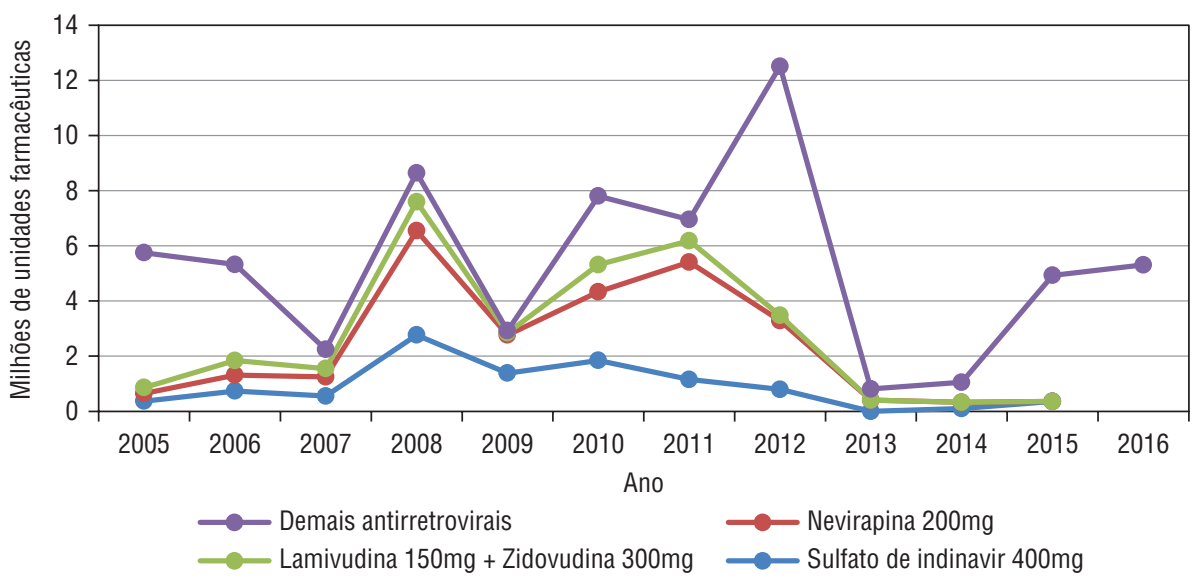

Fonte: Sistema de Gestão de Materiais do Ministério da Saúde (SISMAT).

com disponibilidade constante desses medicamentos; o desenvolvimento do SUS no que se refere ao aperfeiçoamento das ações de assistência farmacêutica e da oferta contínua desses medicamentos; e, finalmente, a produção pública nacional 
de medicamentos antirretrovirais e antimaláricos, conforme diretrizes e prioridades estabelecidas na Política Nacional de Medicamentos (26).

No que tange às ações do Brasil no enfrentamento da Aids, citam-se o importante papel da sociedade civil e do ativismo de movimentos sociais, a implantação de rede de serviços e laboratórios para atendimento das pessoas vivendo com $\mathrm{HIV} /$ Aids, bem como as iniciativas para garantir o acesso aos medicamentos, como a referida Lei 9 313, a licença compulsória do medicamento efavirenz e a produção local de antirretrovirais pelos laboratórios públicos (27). Destaca-se a atuação do Brasil na esfera internacional, como, por exemplo, sua participação ativa na Comissão de Direitos Humanos das Nações Unidas envolvendo aspectos-chave como a defesa do direito do cidadão aos antirretrovirais e também as proposições sobre a flexibilidade dos acordos de patentes envolvendo esses medicamentos $(27,28)$.

Para além da atuação no âmbito de organismos internacionais, a literatura tem relatado que o Brasil vem estabelecendo processos de cooperação bilateral com países africanos e latino-americanos. Tais processos são pautados em princípios da solidariedade e cooperação horizontal, nos quais as doações de medicamentos e transferências de tecnologias poderiam contribuir para a implantação de programas de acesso aos antirretrovirais nos países receptores (27).

Quando comparado a outros países, o Brasil ainda não possui expressiva necessidade de receber medicamentos doados por ajuda humanitária externa, não tendo sido identificada a existência de protocolos ou manuais sobre a gestão de medicamentos recebidos por ajuda humanitária (29). No entanto, cabe apontar que o Brasil recebe doações de medicamentos para três (oncocercose, geo-helmintíases e hanseníase) dos seis agravos incluídos no plano integrado de ações estratégicas de eliminação da hanseníase, filariose, esquistossomose e oncocercose, tracoma e controle das geo-helmintíases (30).

Uma solicitação internacional de medicamentos pode demonstrar fragilidade no acesso e necessita de uma avaliação das necessidades do território com base nos dados epidemiológicos, oferta de serviços e consumo histórico. No entanto, em situação de conflito, desastre ou outro tipo de emergência, ações de preparo possibilitam o enfrentamento dessa situação por governos e sociedade $(15,29)$. Uma programação inadequada de medicamentos pode gerar subutilização, divergência no esquema terapêutico, excesso de medicamentos e perda pelo prazo de validade. Medicamentos doados podem trazer sobrecarga de tempo e esforço, pois precisam ser triados e encaminhados a sua destinação de uso, preservando as condições necessárias de armazenamento e conservação $(29,31)$.

Os países receptores devem ter um sistema estabelecido de descarte, pois, ao receberem os medicamentos doados, precisam estar preparados para eliminar de forma segura e adequada aqueles vencidos ou com avaria (31). Ademais, o gasto logístico pode ser superior ao valor dos medicamentos e, muitas vezes, não é possível contar com abastecimento regular de doações, importante fator sobretudo em casos em que são necessários tratamentos prolongados (20).

Pesquisas (32) apontam a necessidade de que sejam desenvolvidos indicadores de "melhores práticas" para doações farmacêuticas, com compartilhamento das responsabilidades relativas a doação e recebimento, além da implementação de procedimentos para a comunicação regular com os destinatários de uso final, a fim de garantir o máximo de benefício aos países receptores. Toda doação de medicamentos deve obedecer à regulação sanitária (33) e estar atenta para que não haja duplicação de esforço e desperdício de recursos.

Um estudo realizado na Indonésia (34), após a catástrofe do tsunami que devastou a região, relatou como principais inadequações o fato de que $60 \%$ dos medicamentos doados não se encontravam na lista de medicamentos essenciais da Organização Mundial da Saúde (OMS), a rotulagem em língua estrangeira em $70 \%$ e prazo de validade inadequado em $25 \%$. Esse estudo recomendou a inclusão das diretrizes da OMS para doação de medicamentos (35) nas legislações nacionais dos diferentes países, a fim de criar melhores práticas de doações. Essas diretrizes buscam promover a qualidade das doações assistenciais ou emergenciais de medicamentos no campo internacional, com orientações sobre seleção dos medicamentos, embalagem, rotulagem e gestão, além de atribuição de responsabilidades a doadores e receptores. A longo prazo, a independência das intervenções estrangeiras é significativa, em especial nos países periféricos (36). Portanto, é importante que qualquer intervenção de doação de medicamentos seja calcada nos objetivos gerais das diretrizes brasileiras para o desenvolvimento de cooperação técnica internacional, especialmente "atuação mais eficiente e eficaz da instituição brasileira que atuou como executora da iniciativa de cooperação técnica, a partir do aprimoramento de seus processos internos e da elaboração e implementação de planos e estratégias de ação com maior qualidade" (37, p. 7).

Destacam-se como limitações do presente estudo: a falta de registro das informações de doação do Ministério da Saúde antes de 2005, o que poderia influenciar nas análises ora realizadas; a necessidade de desagregar dados primários (por exemplo, quando ocorre registro no SISMAT da menor unidade farmacêutica em blíster, que representa várias unidades farmacêuticas, em vez de comprimido, isso poderia levar a erros no processo de geração, processamento e análise dos dados); e a falta de registro sistemático dos pedidos de doação de medicamentos não atendidos pelo Ministério da Saúde, o que pode levar à viés de informação. Como ponto forte, destaca-se o caráter inédito da caracterização das doações internacionais de medicamentos realizadas pelo Brasil.

\section{CONCLUSÕES}

No âmbito da diplomacia internacional, as doações de medicamentos pelo governo brasileiro podem fortalecer a cooperação, especialmente a cooperação Sul-Sul, o que aumenta a credibilidade do país no contexto humanitário e solidário e pode aliviar efeitos de agendas restritivas oriundas de crises econômicas ou exigências dos ajustes macroeconômicos no setor saúde. Como perspectivas para aprimoramento das ações do Ministério da Saúde no que se refere às doações de medicamentos, aponta-se a necessidade de atualização dos sistemas de informação, a fim de possibilitar o registro completo dos dados e a geração de relatórios detalhados para subsidiar a tomada de decisão no menor tempo possível, dada a urgência dos pedidos de doação. Vale frisar que protocolos-padrão e as ações regulatórias sobre a doação de medicamentos demandam constante avaliação 
e aperfeiçoamento, a fim de que haja eficiência no atendimento de países em crises humanitárias.

Ao descrever o perfil das doações internacionais de medicamentos realizadas pelo Brasil, espera-se que o presente trabalho possa promover reflexões sobre as ações de cooperação em saúde por meio da doação de medicamentos e insumos. ment in health co-operation: what can it contribute to the global health debate? Health Policy Plan. 2014;29(2):266-70.

2. London L, Himonga C, Fick N, Stuttaford M. Social solidarity and the right to health: essential elements for people-centered health systems. Health Policy Plan. 2015;30(7):938-45.

3. Brasil. Lei 9 279/1996. Disponível em: http://legislacao.planalto.gov.br/legisla/ legislacao.nsf/Viw_Identificacao/lei $\% 20$ 9.279-1996?OpenDocument Acessado em março de 2018.

4. United Nations Secretary-General's HighLevel Panel on Access to Medicines. Report of the United Nations Secretary-General's High-Level Panel on Access to Medicines: Promoting innovation and access to health technologies; 2016. Disponível em: http:/ / www.unsgaccessmeds.org/final-report Acessado em 9 de março de 2017.

5. Santana RS, Leite SN. Prioridades da pesquisa clínica com medicamentos no Brasil e as doenças da pobreza. Rev Panam Salud Publica. 2016;40(5):356-62.

6. Brasil, Presidência da República. Decreto 7 540/2011. Disponível em: http://www. planalto.gov.br/ccivil_03/_ato2011-2014/ 2011/decreto/d7540.htm Acessado em março de 2018.

7. Brasil, Ministério da Saúde. Portaria GM/ MS 506/2012. Disponível em: http://bvsms.saude.gov.br/bvs / saudelegis / gm/2012/prt0506_21_03_2012.html Acessado em março de 2018.

8. Brasil, Ministério da Saúde. Portaria GM/ MS 837/2012. Disponível em: http://bvsms.saude.gov.br/bvs/saudelegis/gm/ 2012/prt0837_18_04_2012.html Acessado em março de 2018 .

9. Brasil, Ministério da Saúde. Ciência e tecnologia e Ccmplexo industrial - produtores oficiais. Disponível em: http:// portalms.saude.gov.br/ciencia-e-tecnologia-e-complexo-industrial/complexo-industrial/produtores-oficiais Acessado em 8 de janeiro de 2018.

10. Brasil, Ministério da Saúde. Países economizam até $83 \%$ em primeira compra conjunta de medicamentos. 2015. Disponível em: http://portalsaude.saude.gov.br/index. $\mathrm{php} / \mathrm{cidadao} / \mathrm{principal/agencia-saude/}$ 20764-paises-economizam-ate-83-emprimeira-compra-conjunta-de-medica mentos Acessado em 9 de março de 2017.

11. Brasil, Ministério do Planejamento, Desenvolvimento e Gestão. Plano Plurianual.

Como recomendação para novos estudos, sugerem-se análises comparadas de políticas externas em saúde, especialmente aquelas relacionadas a medicamentos e insumos para saúde no âmbito das cooperações Sul-Sul ou Norte-Sul, que tenham potencial de fomentar o desenvolvimento social e econômico de países receptores.

\section{REFERÊNCIAS}

Disponível em: http:/ /www.planejamento.gov.br/assuntos/planeja/plano-plurianual Acessado em 20 de janeiro de 2018.

12. Fonseca LE, Buss PM. Diplomacia e cooperação em saúde: uma perspectiva da Fiocruz. Em: Brasil. Ministério das Relações Exteriores (MRE). Agência Brasileira de Cooperação (ABC). 30 anos da ABC: visões da cooperação técnica internacional brasileira. Brasília: Fundação Alexandre Gusmão; 2017. Pp. 223-54. Disponível em: http://funag.gov.br/ loja / download/1193-30-anos-ABC_ 05_05_V_7.pdf Acessado em março de 2018.

13. Gomes RMS, Oliveira VC. Cooperação internacional Brasil-Cuba-Haiti: o papel das rádios comunitárias no fortalecimento da mobilização social no âmbito da saúde pública no Haiti. Cien Saude Colet. 2015;20(1):199-208

14. Brasil. Programa de Cooperação Internacional para Ações de Prevenção e Controle do HIV/Aids para outros países em desenvolvimento; 2002. Disponível em: http://bvsms.saude.gov.br/bvs/publicacoes/programa_port.pdf Acessado em 21 de maio de 2017.

15. Fundo das Nações Unidas para a Infância (UNICEF). Rede Laços Sul-Sul. Uma iniciativa internacional de cooperação para o enfrentamento da epidemia HIV/AIDS. Disponível em: http://www.unicef.org/ brazil/pt/rede_sul_sul_pt.PDF Acessado em 13 de fevereiro de 2017.

16. Dunda FFE. A Diplomacia de Nicho brasileira no contexto da Cooperação SulSul em HIV/AIDS com países Africanos durante o Governo Lula. Em: Redefinindo a Diplomacia num Mundo em Transformação. $5^{\circ}$ Encontro Nacional da ABRI. Disponível em: www.encontronacional2015.abri.org.br Acessado em 10 de março de 2017.

17. Santos RF, Cerqueira MR. South-South Cooperation: Brazilian experiences in South America and Africa. Hist Cienc Saude Manguinhos. 2015;22(1):23-47.

18. Almeida EL. Entre o discurso solidário e a ação pragmática da cooperação brasileira em Moçambique: os casos dos projetos de implantação da fábrica de medicamentos antirretrovirais e o ProSavana. Cad CRH. 2016;29(76):53-68.

19. Fedatto MS. The AIDS Epidemic and the Mozambican Society of Medicines: an analysis of Brazilian cooperation. Cien Saude Colet. 2017;22(7):2295-304.
Conflitos de interesse. Nada declarado pelos autores.

Declaração. As opiniões expressas no manuscrito são de responsabilidade exclusiva dos autores e não refletem necessariamente a opinião ou política da RPSP/PAJPH ou da Organização PanAmericana da Saúde (OPAS).

20. Pinheiro PC. Drug donations: what lies beneath. Bull World Health Organ. 2008; 86(8):577-656

21. Brasil, Ministério da Saúde. Portaria 2 069/2003. Disponível em: http: / /bvsms. saude.gov.br/bvs/saudelegis/gm/2003/ prt2069_30_10_2003.html Acessado em março de 2018.

22. Instituto Brasileiro de Geografia e Estatística (IBGE). Índice de Preços ao Consumidor Amplo (IPCA). Disponível em: http://www.ibge.gov.br/home/estatistica/indicadores/precos/inpc_ipca/ defaultinpc.shtm Acessado em 31 de dezembro de 2017.

23. Brasil, Ministério das Relações Exteriores, Agência Brasileira de Cooperação. Manual de Gestão da Cooperação Técnica Sul-Sul. Brasília, Ministério das Relações Exteriores; 2013. Disponível em: http:/ / www.abc.gov. br/content/abc/docs/manual_sulsul final-diagramado_corrigido_23-04-2014. pdf Acessado em 27 de dezembro de 2017.

24. World Health Organization (WHO). Millennium Development Goals (MDGs) Disponível em: http:/ / www.who.int/mediacentre/factsheets/fs290/en/ Acessado em 15 de julho de 2017.

25. Brasil. Lei 9 313/1996. Disponível em: http: / / www.planalto.gov.br/ccivil_03/ Leis/L9313.htm Acessado em março de 2018.

26. Brasil, Ministério da Saúde. Portaria GM/ MS 3 916/1998. Aprova a Política Nacional de Medicamentos. http://bvsms.saude. gov.br/bvs / saudelegis / gm / 1998/ prt3916_30_10_1998.html Acessado em março de 2018.

27. Grangeiro A, Silva LL, Teixeira PR. Resposta à aids no Brasil: contribuições dos movimentos sociais e da reforma sanitária. Rev Panam Salud Publica. 2009; 26(1):87-94.

28. Greco DB. Trinta anos de enfrentamento à epidemia da Aids no Brasil, 1985-2015. Cien Saude Colet. 2016;21(5):1553-64.

29. Miranda ES, Luiza VL, Lima EC, Souza PP, Freitas LF, Osório-de-Castro CG. Como gerir medicamentos em desastres? Orientações básicas. Rio de Janeiro: Fundação Oswaldo Cruz, Escola Nacional de Saúde Pública Sergio Arouca/ Núcleo de Assistência Farmacêutica: 2011. Disponível em: www.informacaoemsaude.rj.gov.br Acessado em 10 de março de 2017.

30. Brasil, Ministério da Saúde. Plano integrado de ações estratégicas de eliminação da 
hanseníase, filariose, esquistossomose e oncocercose, tracoma e controle das geohelmintíases. Disponível em: http://bvsms.saude.gov.br/bvs/publicacoes / plano_integrado_acoes_estrategicas_ 2011_2015.pdf Acessado em 22 de março de 2017.

31. Pimenta-de-Souza P, Miranda ES, Osóriode-Castro CGS. Preparação da assistência farmacêutica para desastres: um estudo em cinco municípios brasileiros. Cien Saude Colet. 2014;19(9):3731-42.

32. Reich MR. An Assessment of US Pharmaceutical Donations: Players, Processes, and Products. Boston: Harvard School of Public Health; 1999. Disponível em: http:// apps.who.int/medicinedocs / en / d / Js20190en/ Acessado em março de 2018.

33. Santos MMFLCS. Enquadramento regulamentar do comércio internacional de medicamentos: o papel das autoridades reguladoras [dissertação]. Lisboa: Universidade de Lisboa; 2010. Disponível em: http:/ / repositorio.ul.pt/handle/10451/ 2654 Acessado em 10 de abril de 2017.

34. Pharmaciens Sans Frontiéres Comité International. Study of drug donations in the province of Aceh in Indonésia. 2005. Disponível em: http://apps.who.int/ medicinedocs / documents / s17066e/ s17066e.pdf Acessado em 13 de abril de 2017.

35. World Health Organization. Guidelines for medicine donations. Revised 2010. Disponível em: http://www.who.int/selection_medicines/emergencies/guidelines medicine_donations/en/ Acessado em 10 de abril de 2017.

36. Igoumenidis M, Kyriopoulos I, Athanasakis K. Drug and Cash Donations to Developing Countries Problems and Proposals. J Dev Soc. 2013;29(3):287-303.

37. Brasil, Ministério das Relações Exteriores, Agência Brasileira de Cooperação. Diretrizes para o desenvolvimento da cooperação técnica internacional multilateral $\mathrm{e}$ bilateral. Brasília: Ministério das Relações Exteriores; 2014. $4^{\mathrm{a}}$ ed. Disponível em: http://www.abc.gov.br/Content/ABC/ docs/ManualDiretrizesCooperacaoRecebida.pdf Acessado em 27 de dezembro de 2017.

Manuscrito recebido em 16 de agosto de 2017. Aceito em versão revisada em 27 de janeiro de 2018.
ABSTRACT

International cooperation: drugs donated by the Brazilian government, 2005-2016

Keywords
Objective. To perform a survey of international drug donations by the Brazilian government from 2005 to 2016, identifying the number of drug units donated and the corresponding financial amount.

Method. This descriptive and exploratory study used secondary data available at the Brazilian Health Ministry's Materials Management System (SISMAT). The following information was retrieved: requesting country, year of donation, drug donated, number or pharmaceutical unit donated, and corresponding amount (in Brazilian reais).

Results. During the study period, 66554892 pharmaceutical units were donated, corresponding to R\$ 84371308.72 (US\$ 25505 235.70). Drugs were donated to Angola, Benin, Bolivia, Burkina Faso, Cape Verde, Colombia, Ivory Coast, Cuba, El Salvador, Ecuador, Guatemala, Guiana, Republic of Guinea-Bissau, Haiti, Honduras, Libya, Mozambique, Nicaragua, Paraguay, Peru, Dominican Republic, Sao Tome and Principe, Syria, and Suriname, countries from the Caribbean Community and Common Market (CARICOM), and Pan-American Health Organization (PAHO). No distribution pattern was detected in quantitative terms. Peaks in the number of drugs donated were recorded in 2008, 2011, and 2012, with the largest number of countries receiving donations in $2006(n=13)$ and $2012(n=14)$. Drugs were donated for the treatment of HIV / Aids, malaria, leishmaniasis, diabetes, cholera, schistosomiasis, tuberculosis, influenza, opportunistic diseases, immunizations, nutrition support, and health conditions associated with natural disasters.

Conclusions. The drugs donated by the Brazilian government included mostly antimicrobial agents used for the treatment of neglected tropical diseases. Further studies are suggested to correlate public health interventions with the donation of drugs as a means to promote the sustainable economic development of the recipient countries. 
RESUMEN Objetivo. Hacer un inventario de las donaciones internacionales de medicamentos del gobierno brasileño entre el 2005 y el 2016, estableciendo el número de unidades de medicamentos donadas y el monto económico que representa ese volumen.

Cooperación internacional: donaciones de medicamentos del gobierno brasileño entre el 2005 y el 2016

Métodos. Estudio descriptivo y exploratorio utilizando análisis de datos secundarios disponibles en el Sistema de Gestión de Materiales del Ministerio de Salud (SISMAT). Se recogió información sobre el país solicitante, año de la donación, medicamento, número de unidades farmacéuticas donadas y valor correspondiente (en reales brasileños).

Resultados. En el período estudiado, se donaron 66554892 unidades de medicamentos, que corresponden a 84371308,72 reales (25505235,70 dólares). Se donaron medicamentos a Angola, Benin, Bolivia, Burkina Faso, Cabo Verde, Colombia, Côte d'Ivoire, Cuba, El Salvador, Ecuador, Guatemala, Guyana, Guinea Bissau, Haití, Honduras, Libia, Mozambique, Nicaragua, Paraguay, Perú, República Dominicana, Santo Tomé y Príncipe, Siria y Suriname, y a otros países miembros del Mercado Común y Comunidad del Caribe (CARICOM) y de la Organización Panamericana de la Salud (OPS). No se ha detectado ningún patrón de distribución en términos cuantitativos. Hubo picos en el número de medicamentos donados en el 2008, el 2011 y el 2012, con un mayor número de países receptores en el 2006 y el 2012 (13 y 14 países, respectivamente). Se donaron medicamentos para la infección por el VIH/sida, la malaria, la leishmaniasis, la diabetes, el cólera, la esquistosomiasis, la tuberculosis, la gripe aviar y las infecciones oportunistas, así como insumos para vacunaciones, apoyo nutricional y desastres.

Conclusiones. La mayoría de los medicamentos donados eran antimicrobianos utilizados para el tratamiento de enfermedades tropicales desatendidas. Se sugiere llevar a cabo estudios adicionales para mostrar la relación entre las intervenciones de salud pública y las donaciones de medicamentos como forma de promover el desarrollo económico sostenible de los países beneficiarios.

Palabras clave Cooperación internacional; donaciones; medicamentos esenciales; prestación de atención de salud; Brasil. 